Originalien

Orthopäde $2021 \cdot 50: 859-865$

https://doi.org/10.1007/s00132-021-04088-7

Angenommen: 11. Februar 2021

Online publiziert: 9. März 2021

๑ Der/die Autor(en) 2021
Nike Walter ${ }^{1,2} \cdot$ Markus Rupp $^{1} \cdot$ Thilo Hinterberger ${ }^{2} \cdot$ Volker Alt $^{1}$

'Klinik und Poliklinik für Unfallchirurgie, Universitätsklinikum Regensburg, Regensburg, Deutschland

${ }^{2}$ Abteilung für Psychosomatische Medizin, Universitätsklinikum Regensburg, Regensburg, Deutschland

\title{
Protheseninfektionen und die zunehmende Bedeutung psychologischer Komorbiditäten
}

\author{
Eine epidemiologische Analyse für \\ Deutschland von 2009 bis 2019
}

\begin{abstract}
Periprothetische Gelenkinfektionen (PJI) sind eine gefürchtete Komplikation in der Orthopädie und Unfallchirurgie. Ein potenzieller Anstieg an PJI-Diagnosen, insbesondere in Verbindung mit psychologischen Komorbiditäten, kann zu einer besonderen Herausforderung für Akteure im Gesundheitswesen werden. Bisher ist die Prävalenz für Deutschland unbekannt. Dies erschwert es, den zukünftigen Behandlungsbedarf abzuschätzen und Entwicklungen vorherzusehen, die durch eine Anpassung von Präventions- und Therapiemaßnahmen beeinflusst werden können. In diesem Beitrag wird eine epidemiologische Analyse berichtet.
\end{abstract}

\section{Hintergrund und Fragestellung}

Der endoprothetische Gelenkersatz ist ein lebensverbesserndes Verfahren für Millionen von Menschen auf der ganzen Welt, der neben einer Schmerzlinderung auch die Funktion des betroffenen Gelenkes wiederherstellen kann. In Deutschland gehört das Implantieren einer Knie- oder Hüftendoprothese zu den häufigsten Eingriffen, wobei ein Anstieg der Operationszahlen um bis zu $45 \%$ für das Jahr 2040 prognostiziert wurde [24]. Eine gefürchtete Komplikation in der Orthopädie und Unfallchirurgie ist die periprothetische Gelenkinfektion (PJI). Für Patienten stellt eine Protheseninfektion eine hohe Belastung dar und schwer- wiegende Einschränkungen sind oft trotz modernster und interdisziplinärer Behandlungskonzepte - nicht zu vermeiden. Folgen sind lange Krankenhausaufenthalte mit mehrfachen Operationen, einschließlich Entfernung des Implantats und Resektion des infizierten Knochens, Immobilität, erhöhte Gabe von lokalen und systemischen Antibiotika, häufig verbunden mit Nebenwirkungen und weitere sozioökonomischen Folgen. Darüber hinaus ist eine PJI potenziell lebensbedrohlich mit einer 3,2-3,7fach erhöhten Sterblichkeitsrate im Vergleich zu Patienten, bei denen im Verlauf eines Gelenkersatzes keine Infektion auftritt [26]. Die Behandlung ist komplex und abhängig von diversen Faktoren, wie Dauer der Infektion, zugrundeliegende Erreger sowie Zustand der implantierten Prothese und der Weichteile. Mögliche Optionen sind das chirurgische Debridement mit Implantaterhalt und Antibiotikatherapie, eine einzeitige Revision oder ein zweizeitiger Prothesenwechsel, bei dem eine neue Revisionsendoprothese nach einem implantatfreien Intervall eingesetzt wird. Bei einer beträchtlichen Anzahl von Patienten wird die Reimplantation nicht als durchführbar erachtet, sodass nur eine Gelenkversteifung durch knöcherne Fusion oder ein Arthrodeseimplantant, die Schaffung einer Girdlestone-Situation, sowie die Amputation der affektierten Gliedmaßen als Therapiealternativen verbleiben.

Durch die schlechteren Behandlungsergebnisse im Vergleich zu Fällen, die keine Revision benötigen, haben PJI, verbunden mit Schmerzen, Bewegungseinschränkungen sowie Verlust der Mobilität und Selbstständigkeit, tiefgreifende Auswirkungen auf die Lebensqualität betroffener Patienten und führen zu einem hohen Maß an psychologischem Stress [7, 9, 16, 17, 23, 32].

Durch die Zunahme der Primärimplantationen und PJI-spezifischen Risikofaktoren wie Übergewicht oder Diabetes mellitus kann ebenfalls ein erhöhtes Auftreten von Gelenksinfektionen erwartet werden $[8,15,30]$. Während ansteigende PJI-Inzidenzen für die USA, Dänemark, Finnland, Norwegen, Schweden, Neuseeland, England und Irland beschrieben wurden [4, 12, 27], ist die Prävalenz für Deutschland unbekannt. Dieses erschwert es, den zukünftigen Bedarf abzuschätzen und Entwicklungen vorherzusehen, die durch eine Anpassung der Präventions- und Therapiemaßnahmen beeinflusst werden könnten. Neben weiteren somatischen Nebenerkrankungen, wie rheumatoide Arthritis und Hyperthyreose [10, 29], die das Auftreten einer Infektion

\begin{tabular}{|ll}
\hline Abkürzungen \\
\hline COVID-19 & "Coronavirus disease 2019“ \\
\hline G-DRG & German Diagnosis Related Groups \\
\hline ICD & $\begin{array}{l}\text { International Statistical Classifi- } \\
\text { cation of Diseases and Related } \\
\text { Health Problems }\end{array}$ \\
\hline PJI & Periprothetische Gelenkinfektion \\
\hline
\end{tabular}


Tab. 1 Beschreibung der verwendeten ICD-10-Diagnoseschlüssel

ICD- Beschreibung Beispielerkrankungen

10-

Kode

T84.5 Infektion und entzündliche Reak- Periimplantäre (implantatassoziierte) Infektion tion durch eine Gelenkendoprothese

F0 Organische, einschließlich sym- Demenz bei Alzheimer-Krankheit, vaskuläre Demenz, ptomatischer psychischer Störun- Delir, nicht durch Alkohol oder andere psychotrope gen Substanzen bedingt

F1 Psychische und Verhaltensstörun- Psychische und Verhaltensstörungen durch Alkohol, gen durch psychotrope Substan- Cannabinoide, Tabak, Sedativa oder Hypnotika zen

F2 Schizophrenie, schizotype und wahnhafte Störungen

F3 Affektive Störungen

F4 Neurotische, Belastungs- und somatoforme Störungen

F5 Verhaltensauffälligkeiten mit körperlichen Störungen und Faktoren

F6 Persönlichkeits- und Verhaltensstörungen

F7 Intelligenzstörungen

F8 Entwicklungsstörungen

F9

Verhaltens- und emotionale Störungen mit Beginn in der Kindheit und Jugend
Schizophrenie, schizotype Störung, anhaltende wahnhafte Störung

Manische Episode, bipolare affektive Störung, depressive Episode

Phobische Störungen, Zwangsstörungen, dissoziative Störungen

Essstörungen, nichtorganische Schlafstörungen

Spezifische Persönlichkeitsstörungen: paranoide, dissoziale, emotional instabile (impulsiver Typ, Borderline-Typ)

Leichte Intelligenzminderung, schwere Intelligenzminderung

Umschriebene Entwicklungsstörungen des Sprechens und der Sprache, umschriebene Entwicklungsstörungen motorischer Funktionen

Hyperkinetische Störungen, Ticstörungen beeinflussen, spielen auch psychologische Komorbiditäten eine entscheidende Rolle. So erhöht beispielsweise Tabakkonsum, bei Abhängigkeitssyndrom und schädlichem Gebrauch kodiert unter Kapitel F17 des ICD-10, das Infektionsrisiko nach Gelenkersatz signifikant [5]. Auch die Nebendiagnose Depression führt zu erhöhtem Infektrisiko nach Gelenkersatz und ist assoziiert mit schlechteren klinischen Ergebnissen [2, 3, 22], wobei jedoch bisher das Potenzial von psychologisch unterstützenden Interventionen nicht untersucht wurde [11].

Daher ist das Ziel der vorliegenden Arbeit die Beantwortung der folgenden Forschungsfragen:

- Wie haben sich die PJI-Inzidenzen im Laufe des letzten Jahrzehnts entwickelt?

- Welchen Einfluss haben die Faktoren Alter und Geschlecht auf die Häufigkeit der Diagnose?
- Wie hat sich der Anteil an Patienten mit psychologischen Nebenerkranken in den letzten 10 Jahren entwickelt?

- Welche psychologischen Komorbiditäten kommen am häufigsten bei PJI vor?

\section{Studiendesign und Unter- suchungsmethoden}

Ein Datenset, bestehend aus jährlichen ICD-10-Diagnosekodes aus deutschen medizinischen Einrichtungen von 2009 bis 2019, wurden vom Statistischen Bundesamt (Destatis) zur Verfügung gestellt. Zunächst wurde die Anzahl der ICD10-Diagnosen mit dem Kode „T84.5 Infektion und entzündliche Reaktion durch eine Gelenkendoprothese " nach Altersgruppe in 10-Jahres-Inkrementen und Geschlecht quantifiziert. Die Prävalenzraten wurden auf Basis der von Destatis bereitgestellten histori- schen Bevölkerung Deutschlands älter als 20 Jahre berechnet [28]. Dabei wurde für jedes Jahr des Zeitraums 2009 bis 2019 die Anzahl der Einwohner in jedem der 16 Bundesländer nach Geburtsjahrgängen betrachtet. Stichtag eines jeden Jahres war der 31. Dezember. Zusätzlich wurde die Anzahl der stationären $\mathrm{Pa}$ tienten mit der Hauptdiagnose „T84.5“ und einer Nebendiagnose des Kapitels F des ICD-10, Psychische und Verhaltensstörungen, bestimmt. Die prozentuale Verteilung der Nebendiagnosen wurden unterteilt für die Diagnosen „F0, F1, F2, F3, F4, F5, F6, F7, F8“ und „F9“(• Tab. 1). Darüberhinausgehende, weitere Nebendiagnosen wurden in der Auswertung nicht berücksichtigt.

\section{Ergebnisse}

Seit dem Jahr 2009 konnte ein kontinuierlicher Anstieg der PJI-Diagnosen verzeichnet werden, die mit 18.797 Fällen im Jahr 2017 ein Maximum erreichten, woraufhin die Häufigkeit abfiel auf 16.174 Fälle im Jahr 2019 mit einer Inzidenz von 23,8/100.000 Einwohner. Im Vergleich zum Jahr 2009 betrug die relative Veränderung $+3,54 \%$ (• Tab. 2). Die Geschlechterverteilung blieb über das letzte Jahrzehnt nahezu konstant. Das FrauenMänner-Verhältnis betrug 52,1/47,9\% im Jahr 2009 bzw. 51,3/48,7\% im Jahr 2019. Im Jahr 2019 waren Frauen im Alter von 70-79 Jahren am häufigsten betroffen (17,64\%), gefolgt von der Altersgruppe 80-89 Jahre (15,35\%) und 60-69 Jahre (10,59\%). Der größte Anteil der PJIDiagnosen bei Männern im Jahr 2019 wurde für die Altersgruppe 70-79 Jahre berechnet $(16,61 \%)$, gefolgt von Patienten im Alter 60-69 Jahren (12,96\%) und 80-89 Jahren (9,9\%) (• Abb. 1). Insgesamt konnte eine Entwicklung hinsichtlich mehr PJI-Diagnosen bei älteren Patienten beobachtet werden, mit einem Anstieg von 25,70\% der Fälle in der Altersgruppe 80-89 Jahre bei Frauen und $+71,24 \%$ Veränderung bei Männern im Alter 80-89 Jahre zwischen 2009 und 2019. Die Fallzahlen für das Altersinkrement älter als 90 Jahre stiegen für Frauen ums 2,3fache, bei Männern gab es eine 2,8fache Zunahme. 
Orthopäde 2021·50:859-865 https://doi.org/10.1007/s00132-021-04088-7

(c) Der/die Autor(en) 2021

N. Walter · M. Rupp · T. Hinterberger · V. Alt

\section{Protheseninfektionen und die zunehmende Bedeutung psychologischer Komorbiditäten. Eine epidemiologische Analyse für Deutschland von 2009 bis 2019}

\section{Zusammenfassung}

Hintergrund. Die periprothetische Ge-

lenkinfektion (PJI) ist eine gefürchtete

Komplikation in der Orthopädie und

Unfallchirurgie. Ein potenzieller Anstieg an

PJI-Diagnosen, insbesondere in Verbindung

mit psychologischen Komorbiditäten, kann

zu einer besonderen Herausforderung für

Akteure im Gesundheitswesen werden. Bisher

ist die Prävalenz für Deutschland unbekannt.

Dies erschwert es, den zukünftigen Behand-

lungsbedarf abzuschätzen und Entwicklungen vorherzusehen, die durch eine Anpassung von Präventions- und Therapiemaßnahmen beeinflusst werden können.

Ziel der Arbeit. Die vorliegende Arbeit gibt eine detaillierte Übersicht über die Epidemio- logie periprothetischer Gelenkinfektionen und psychologischer Komorbiditäten. Material und Methoden. Ein Datensatz vom Statistischen Bundesamt (Destatis) aus jährlichen, deutschlandweiten ICD-10Diagnosekodes von 2009 bis 2019 wurde analysiert. Prävalenzraten des Kodes „T84.5 Infektion und entzündliche Reaktion durch eine Gelenkendoprothese" wurden nach Altersgruppe, Geschlecht und in Verbindung mit einer Nebendiagnose des Kapitels $F$ quantifiziert und aufgeschlüsselt. Ergebnisse. Seit 2009 steigen die PJIDiagnosen kontinuierlich an, die Häufigkeit war im Jahr 2018 rückläufig. Im Jahr 2019 wurden 16.174 Fälle entsprechend einer Prävalenz von 23,8/100.000 Einwohner verzeichnet. Eine Entwicklung hinsichtlich mehr Diagnosen bei älteren Patienten wurde evident. Ein Viertel aller Patienten wiesen eine Nebendiagnosen im Bereich psychischer Störungen und Verhaltensstörungen auf, wobei sich die Anzahl an Patienten mit psychologischen Komorbiditäten im letzten Jahrzehnt verdoppelte.

Schlussfolgerung. Richtlinien zu Präventionsstrategien und psychologische Unterstützungsangebote sollten in der Unfallchirurgie implementiert werden.

Schlüsselwörter Periprothetische Gelenkinfektion · Psychologische Symptome · Epidemiologie Nebenerkrankungen · Gelenkersatz

\section{Prosthetic infections and the increasing importance of psychological comorbidities. An epidemiological analysis for Germany from 2009 through 2019}

\section{Abstract}

Background. Periprosthetic joint infection (PJI) is a devastating complication in orthopedics and trauma surgery. A potential increase in PJI diagnoses, especially in combination with psychological comorbidities may become a significant challenge for healthcare stakeholders. So far, the prevalence for Germany is unknown, which makes it difficult to estimate future treatment demand or to predict developments that can be influenced by adapting prevention and treatment measures.

Objectives. The present study provides a detailed overview of the epidemiology of periprosthetic joint infections and psychological comorbidities.

\begin{abstract}
Materials and methods. A dataset provided by the Federal Statistical Office (Destatis) consisting of annual, Germany-wide ICD10 diagnosis codes from 2009 through 2019 was analyzed. Incidences of the code "T84.5-Infection and inflammatory reaction due to joint arthroplasty" were quantified, and a detailed breakdown of this data by age group and gender was performed. Prevalence rates of secondary diagnoses of the ICD-10 chapter $\mathrm{F}$ were determined.

Results. PJI diagnoses have been steadily increasing since 2009. The incidence declined in 2018. In 2019, 16,174 cases were registered, corresponding to a prevalence of $23.8 / 100,000$ population. A trend towards more diagnoses
\end{abstract}

in elderly patients became evident. Twentyfive percent of all patients had a secondary diagnosis regarding mental and behavioural disorders. The number of patients with psychological comorbidities doubled in the last decade.

Conclusions. Guidelines on prevention strategies as well as psychological support services should be implemented in trauma surgery.

\section{Keywords}

Periprosthetic joint infection · Psychological symptoms - Epidemiology · Comorbidities . Total joint arthroplasty
Im Jahr 2019 wurden $25 \%$ der PJIPatienten mit einer psychischen Nebenerkrankung registriert. Die Häufigkeit der Kapitel-F-Nebendiagnosen stieg seit 2009 stetig an, mit einer Zunahme von $42,1 \%$ von 2846 Fälle auf 4043 Fälle im Jahr 2019 (• Tab. 2; • Abb. 2). Insbesondere die Nebendiagnose F0, organische, einschließlich symptomatischer psychischer Störungen, erhöhte sich in den letzten 10 Jahren um $88,05 \%$ und betraf mit 1589 Fällen im Jahr 2019 39,3 \% aller PJI-
Patienten mit psychologischen Komorbiditäten. Ein Anstieg wurde ebenfalls bezüglich des ICD-Kodes F3, affektive Störungen, verzeichnet (+43,29\%). Mit 1473 Fällen im Jahre 2019 waren 36,43 \% der Patienten mit Nebendiagnosen betroffen. Am dritthäufigsten kamen psychische und Verhaltensstörungen durch psychotrope Substanzen (F1) mit 13,13\% und 513 Fällen im Jahr 2019 bei PJI-Patienten vor (• Tab. 3; • Abb. 3).

\section{Diskussion}

In dieser Studie wurde die Häufigkeit von periprothetischen Gelenkinfektionen in Verbindung mit psychologischen Komorbiditäten bestimmt. Die deutlich geringere Anzahl der Fälle im Jahr 2019 im Vergleich $\mathrm{zu}$ den Vorjahren, lässt darauf schließen, dass vereinheitlichte Richtlinien zu Präventionsstrategien wirksam werden [21, 25]. Ein Großteil der Fälle war in der Altersgruppe 
Tab. 2 Entwicklung der Prävalenz und Inzidenz von PJI-Diagnosen in Kombination mit Häufigkeiten psychologischer und psychiatrischer Nebendiagnosen für die Jahre 2009 bis 2019

\begin{tabular}{|l|l|l|l|l|l|l|}
\hline Jahr & $\begin{array}{l}\text { PJI-Dia- } \\
\text { gnosen }\end{array}$ & $\begin{array}{l}\text { Relative Veränderung } \\
\text { zu 2009 }\end{array}$ & $\begin{array}{l}\text { Deutsche Population älter als } \\
\text { 20 Jahre }\end{array}$ & $\begin{array}{l}\text { PJI-Inzidenz/100.000 } \\
\text { Einwohner }\end{array}$ & $\begin{array}{l}\text { Nebendiagnosen } \\
\text { Kapitel F }\end{array}$ & $\begin{array}{l}\text { Nebendiagnosen } \\
\text { prozentual }\end{array}$ \\
\hline 2009 & 15.621 & - & 66.400 .066 & 23,5 & 2846 & 18,22 \\
\hline 2010 & 16.676 & $+6,75$ & 66.549 .975 & 25,1 & 3337 & 20,01 \\
\hline 2011 & 17.267 & $+10,54$ & 65.398 .514 & 26,4 & 3517 & 20,37 \\
\hline 2012 & 17.537 & $+12,27$ & 65.665 .069 & 26,7 & 3595 & 20,50 \\
\hline 2013 & 18.124 & $+16,02$ & 65.943 .867 & 27,5 & 3924 & 21,65 \\
\hline 2014 & 18.417 & $+17,90$ & 66.677 .665 & 27,6 & 4005 & 21,75 \\
\hline 2015 & 18.613 & $+19,15$ & 67.097 .676 & 27,7 & 4198 & 22,55 \\
\hline 2016 & 18.543 & $+18,71$ & 67.440 .230 & 27,5 & 4418 & 23,83 \\
\hline 2017 & 18.797 & $+20,33$ & 67.540 .025 & 27,8 & 4495 & 23,91 \\
\hline 2018 & 18.283 & $+17,04$ & 67.724 .921 & 27,0 & 4316 & 23,61 \\
2019 & 16.174 & $+3,54$ & 67.864 .036 & 23,8 & 4043 & 25,00 \\
\end{tabular}

Tab. 3 Historische Entwicklung und prozentuale Häufigkeiten aufgeschlüsselter psychologischer Nebendiagnosen bei PJIPatienten

\begin{tabular}{|l|l|l|}
$\begin{array}{l}\text { Dia- } \\
\text { gnose }\end{array}$ & $\begin{array}{l}\text { Prozentuale } \\
\text { Häufigkeit }\end{array}$ & $\begin{array}{l}\text { Relative Verän- } \\
\text { derung zu 2009 }\end{array}$ \\
\hline F0 & 39,30 & $+88,05$ \\
\hline F1 & 13,13 & $-14,49$ \\
\hline F2 & 2,25 & $+85,71$ \\
\hline F3 & 36,43 & $+43,29$ \\
\hline F4 & 7,62 & $+57,95$ \\
F5 & 0,40 & $-65,22$ \\
\hline F6 & 0,40 & $-48,39$ \\
\hline F7 & 0,40 & $-20,00$ \\
\hline F8 & 0,0 & 0,0 \\
\hline F9 & 0,07 & $-72,73$ \\
\hline
\end{tabular}

70-79 Jahre verzeichnet, wobei eine Entwicklung hinsichtlich mehr PJI-Diagnosen bei älteren Patienten evident wurde. Frauen und Männer waren nahezu gleich verteilt betroffen. Ein Viertel aller Patienten wiesen eine Nebendiagnosen im Bereich psychischer Störungen und Verhaltensstörungen, Kapitel F des ICD10, auf, wobei organische, einschließlich symptomatischer psychischer Störungen und affektive Störungen am häufigsten auftraten. Die Anzahl an Patienten mit psychologischen Komorbiditäten verdoppelte sich im letzten Jahrzehnt. Da die Einführung des G-DRG-Systems bereits im Jahr 2004 umgesetzt wurde, kann der Einfluss im Sinne erhöhter Fallzahlkodierungen als gering erachtet werden. Anhand des analysierten Datensatzes kann keine Aussage über die Behandlungsstrategie getroffen werden.
Unklar ist daher, ob PJI-Patienten mit Nebendiagnosen psychologische Betreuung erhielten. Nichtsdestotrotz spricht der Anstieg für eine Implementierung von psychologischen Screenings und den Einsatz von Fragebögen, wie etwa einem zeitsparenden ICD-10-basierten Symptomrating [31] sowie psychischer Unterstützungsangebote in der Orthopädie und Unfallchirurgie. Obwohl der Bedarf an psychologischer Unterstützung explizit von Patientenseite benannt wurde [13, 17], ist das mentale Wohlbefinden der Patienten bisher kaum in den Fokus der Chirurgie gerückt. Wissenschaftlich ist die Erkenntnis, dass die psychische Verfassung einen signifikanten Einfluss auf das Behandlungsergebnis hat, gut fundiert $[2,6$, $20,22]$. Nichtsdestotrotz wurde in einer systematischen Literaturarbeit, in der 4213 Artikel zur Behandlung von PJI identifiziert wurden, keine Studie gefunden, die Auswirkungen psychologischer Interventionen untersuchten [11]. Wenn eine bessere Versorgung unserer Patienten erreicht werden soll, ist interdisziplinäre Zusammenarbeit geboten und Psychologen oder Psychiater sollten nicht im Behandlungsteam fehlen [14]. Gerade unter den aktuellen Umständen der COVID-19-Pandemie ist mit einer weiteren Zunahme psychischer Erkrankungen zu rechnen. So berichteten Ohliger und Kollegen von einer höheren Prävalenz psychiatrischer Erkrankungen bei orthopädischen Patienten während der COVID-19-Pandemie (76 von 298 Patienten [26\%] vs. 110 von 255 Patienten [43\%], $p<0,001)$ im Vergleich zu 2019 in den USA [19]. Darüber hinaus betonten mehrere Studien ein geringeres psychisches Wohlbefinden in der Allgemeinbevölkerung im Vergleich zur Zeit vor COVID-19 sowie eine erhöhte Symptombelastung bei Patienten mit vorbestehenden psychiatrischen Erkrankungen [1, 33]. Da mit langanhaltenden Belastungen gerechnet werden muss, ist es dringend erforderlich, die Förderung der psychischen Gesundheit und die Prävention nicht nur in der Orthopädie und Unfallchirurgie, sondern auch global zu verstärken [18]. Konkrete Möglichkeiten zur Verbesserung der Lebensqualität beinhalten die Implementierung von psychologischen Beratungsstellen, Rauchentwöhnungsprogrammen und Ernährungsberatung. Ebenfalls sollten mehr Ressourcen für Sozialdienste in Kliniken zur Verfügung gestellt werden, damit Patienten persönlich betreut werden können und auch Angehörige adäquate Orientierungshilfe bekommen. Angebote wie Selbsthilfegruppe, Gesprächskreise und Kurse zu Entspannungsverfahren sollten verstärkt bereitgestellt und Patienten umfassend über diese informiert werden.

\section{Fazit für die Praxis}

\section{- Ein Screening nach psychologischen Symptomen sollte in der Orthopädie und Unfallchirurgie implementiert werden. \\ - Die interdisziplinäre Zusammenar- beit mit Psychiatern wird empfohlen.}




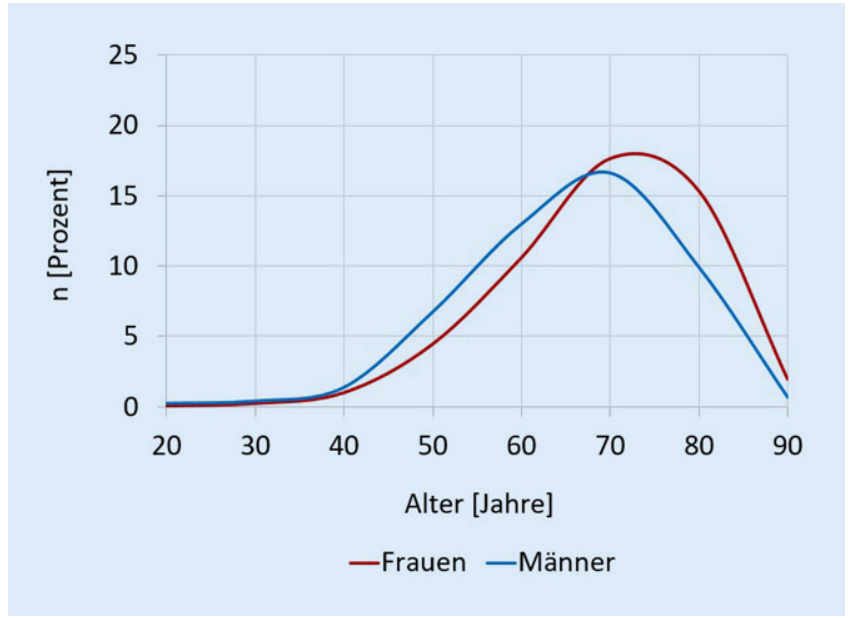

Abb. $1 \triangleleft$ Altersverteilung der Patienten mit diagnostizierter periprothetischer Gelenkinfektion, unterteilt nach Geschlecht, für das Jahr 2019

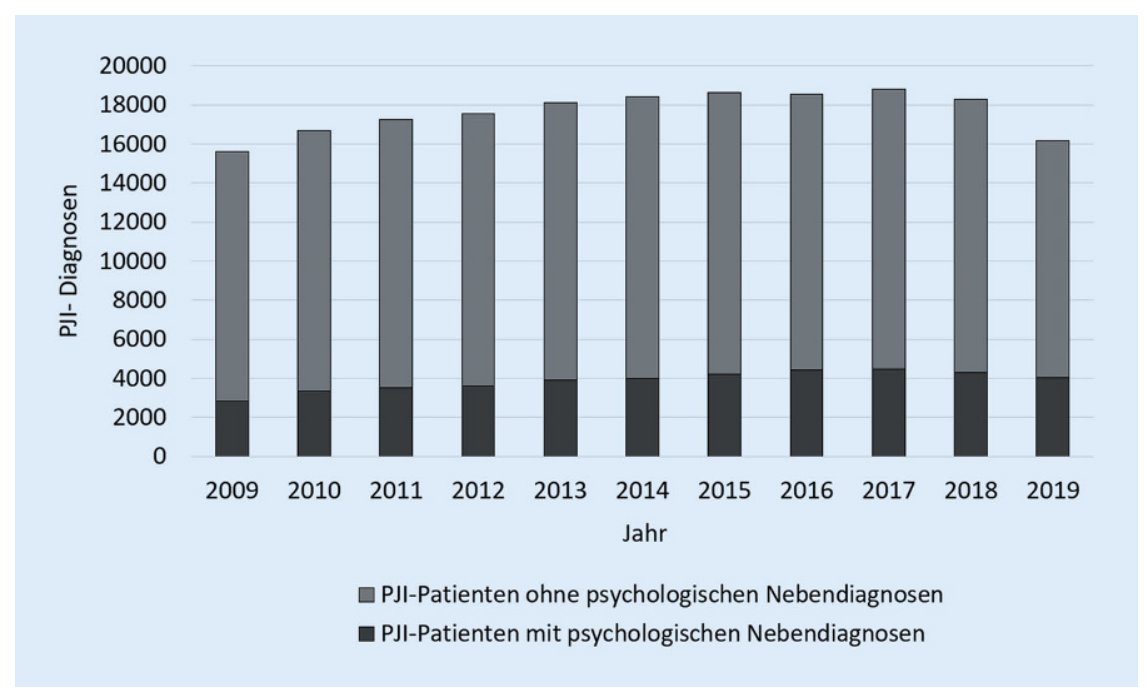

Abb. 2 A Entwicklung der Prävalenz von Diagnosen einer periprothetischen Gelenkinfektion (PJI) über die Jahre 2009 bis 2019. Die Anzahl der Patienten ohne psychologische Nebendiagnosen ist in hellgrau und die Häufigkeit der Patienten mit psychologischen Komorbiditäten in dunkelgrau dargestellt

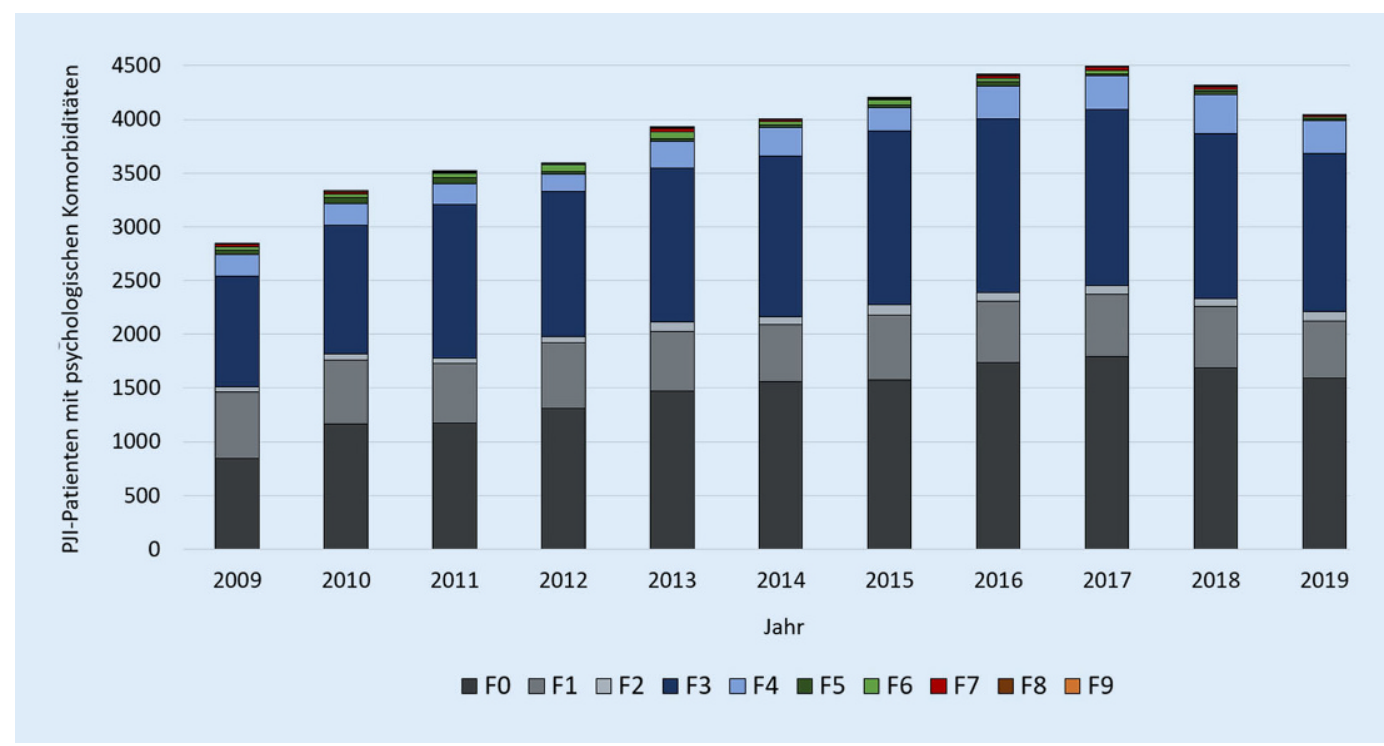

Abb. $3 \triangleleft$ Entwicklung der Prävalenz von Diagnosen einer periprothetischen Gelenkinfektion (PJI) in Verbindung mit psychologischen Komorbiditäten, unterteilt für die Abschnitte des Kapitels F des ICD10 , über die Jahre 2009 bis 2019 


\section{- Psychologische Unterstützungs- angebote zur Verbesserung der Lebensqualität von Patienten mit periprothetischen Gelenkinfektionen werden benötigt}

\begin{tabular}{l} 
Korrespondenzadresse \\
$\begin{array}{l}\text { Prof. Dr. Volker Alt } \\
\text { Kür Unf und Poliklinik } \\
\text { für Unirurgie, } \\
\text { Universitätsklinikum } \\
\text { Regensburg } \\
\text { Franz-Josef-Strauß-Allee 11, } \\
\text { 93053 Regensburg, } \\
\text { Deutschland } \\
\text { volker.alt@ukr.de }\end{array}$ \\
\hline
\end{tabular}

Funding. Open Access funding enabled and organized by Projekt DEAL.

\section{Einhaltung ethischer Richtlinien}

Interessenkonflikt. N. Walter, M. Rupp, T. Hinterberger und V. Alt geben an, dass kein Interessenkonflikt besteht.

Für diesen Beitrag wurden von den Autoren keine Studien an Menschen oder Tieren durchgeführt. Für die aufgeführten Studien gelten die jeweils dort angegebenen ethischen Richtlinien.

Open Access. Dieser Artikel wird unter der Creative Commons Namensnennung 4.0 International Lizenz veröffentlicht, welche die Nutzung, Vervielfältigung, Bearbeitung, Verbreitung und Wiedergabe in jeglichem Medium und Format erlaubt, sofern Sie den/die ursprünglichen Autor(en) und die Quelle ordnungsgemäß nennen, einen Link zur Creative Commons Lizenz beifügen und angeben, ob Änderungen vorgenommen wurden.

Die in diesem Artikel enthaltenen Bilder und sonstiges Drittmaterial unterliegen ebenfalls der genannten Creative Commons Lizenz, sofern sich aus der Abbildungslegende nichts anderes ergibt. Sofern das betreffende Material nicht unter der genannten Creative Commons Lizenz steht und die betreffende Handlung nicht nach gesetzlichen Vorschriften erlaubt ist, ist für die oben aufgeführten Weiterverwendungen des Materials die Einwilligung des jeweiligen Rechteinhabers einzuholen.

Weitere Details zur Lizenz entnehmen Sie bitte der Lizenzinformation auf http://creativecommons.org/ licenses/by/4.0/deed.de.

\section{Literatur}

1. Benros VNME (2020) COVID-19 pandemic and mental health consequences: Systematic review of the current evidence. Brain Behav Immun 89:531-542

2. Browne JA, Sandberg BF, D'Apuzzo MR, Novicoff WM (2014) Depression is associated with early postoperative outcomes following total joint arthroplasty: a nationwide database study. J Arthroplasty 29(3):481-483

3. Buller LT, Best MJ, Klika AK, Barsoum WK (2015) The influence of psychiatric comorbidity on perioperative outcomes following primary total hip and knee arthroplasty; a 17-year analysis of the National Hospital Discharge Survey database. J Arthroplasty 30(2):165-170

4. Dale H, Fenstad AM, Hallan G, Havelin LI, Furnes O, Overgaard Setal (2012) Increasing risk of prosthetic joint infection after total hip arthroplasty. Acta Orthop 83(5):449-458

5. Gonzalez Al, Luime JJ, Uçkay I, Hannouche D, Hoffmeyer P, Lübbeke A (2018) Is there an association between smoking status and prosthetic joint infection after primary total joint Arthroplasty? J Arthroplasty 33(7):2218-2224. https://doi.org/ 10.1016/j.arth.2018.02.069

6. Gouin JP, Kiecolt-Glaser JK (2011) The impact of psychological stress on wound healing: methods and mechanisms. Immunol Allergy Clin North Am 31(1):81-93

7. Helwig $P$, Morlock J, Oberst M, Hauschild $O$, Hübner J, Borde J et al (2014) Periprosthetic joint infection-effect on quality of life. Int Orthop 38(5):1077-1081

8. Khan MAB, Hashim MJ, King JK, Govender RD, Mustafa H, Al Kaabi J (2020) Epidemiology of type 2 diabetes-global burden of disease and forecasted trends. J Epidemiol Glob Health 10(1):107-111

9. Knebel C, Menzemer J, Pohlig F, Herschbach P, Burgkart R, Obermeier Aetal (2020) Peri-prosthetic joint infection of the knee causes high levels of psychosocial distress: a prospective cohort study. Surg Infect 21(10):877-883

10. Kunutsor SK, Whitehouse MR, Blom AW, Beswick AD, Team INFORM (2016) Patient-related risk factors for periprosthetic joint infection after total joint Arthroplasty: a systematic review and metaanalysis. PLoS ONE 11(3):e150866. https://doi.org/ 10.1371/journal.pone.0150866

11. Kunutsor SK, Beswick AD, Peters TJ, GoobermanHill R, Whitehouse MR, Blom AW et al (2017) Health care needs and support for patients undergoing treatment for prosthetic joint infection following hip or knee arthroplasty: a systematic review. PLoSONE 12(1):e169068. https://doi.org/10.1371/ journal.pone. 0169068

12. Kurtz SM, Lau E, Watson H, Schmier JK, Parvizi J (2012) Economic burden of periprosthetic joint infection in the United States. J Arthroplasty 27(8):61-65.e1. https://doi.org/10.1016/j.arth 2012.02.022

13. Mallon CM, Gooberman-Hill R, Moore AJ (2018) Infection after knee replacement: a qualitative study of impact of periprosthetic knee infection. BMC Musculoskelet Disord 19(1):352. https://doi. org/10.1186/s12891-018-2264-7

14. McQueen M (2016) Psychological distress and orthopaedic trauma. J Bone Joint Surg Am 98(5):e19. https://doi.org/10.2106/JBJS.15.01261

15. Mensink GB, Schienkiewitz A, Haftenberger M, Lampert T, Ziese T, Scheidt-Nave C (2013) Übergewicht und Adipositas in Deutschland: ErgebnissederStudiezurGesundheitErwachsener in Deutschland (DEGS1) [Overweight and obesity in Germany: results of the German Health Interview and Examination Survey for Adults (DEGS1)]. Bundesgesundheitsblatt Gesundheitsforschung Gesundheitsschutz 56(5-6):786-794

16. Mur I, Jordán M, Rivera A, Pomar V, González JC, López-Contreras J et al (2020) Do prosthetic joint infections worsen the functional ambulatory outcome of patients with joint replacements? A retrospective matched cohort study. Antibiotics 9(12):872. https://doi.org/10.3390/ antibiotics9120872

17. Moore AJ, Blom AW, Whitehouse MR, GoobermanHill R (2015) Deep prosthetic joint infection: a qualitative study of the impact on patients and their experiences of revision surgery. Bmj Open 5(12):e9495. https://doi.org/10.1136/bmjopen2015-009495

18. Moreno C, Wykes T, Galderisi S, Nordentoft M, Crossley N, Jones $\mathrm{N}$ et al (2020) How mental health care should change as a consequence of the COVID-19 pandemic. Lancet Psychiatry 7(9):813-824. https://doi.org/10.1016/S22150366(20)30307-2

19. Ohliger E, Umpierrez E, Buehler L, Ohliger AW, Magister S, Vallier H et al (2020) Mental health of orthopaedic trauma patients during the 2020 COVID-19 pandemic. IntOrthop 44(10):1921-1925

20. Pan X, Wang J, Lin Z, Dai W, Shi Z (2019) Depression and anxiety are risk factors for postoperative painrelated symptoms and complications in patients undergoing primary total knee Arthroplasty in the United States. J Arthroplasty 34(10):2337-2346 https://doi.org/10.1016/j.arth.2019.05.035

21. Parvizi J, Shohat N, Gehrke T (2017) Prevention of periprosthetic joint infection: new guidelines. Bone Joint J 99-B(4 Supple B):3-10. https://doi. org/10.1302/0301-620X.99B4.BJJ-2016-1212.R1

22. Rasouli MR, Menendez ME, Sayadipour A, Purtill JJ, Parvizi J (2016) Direct cost and complications associated with total joint Arthroplasty in patients with preoperative anxiety anddepression. J Arthroplasty 31(2):533-536. https://doi.org/10. 1016/j.arth.2015.09.015

23. Rietbergen L, Kuiper JW, Walgrave S, Hak L, Colen S (2016) Quality of life after staged revision for infected total hip arthroplasty: a systematic review. Hip Int 26(4):311-318. https://doi.org/10.5301/ hipint.5000416

24. Rupp M, Lau E, Kurtz SM, Alt V (2020) Projections of primary TKA and THA in Germany from 2016 through 2040. Clin Orthop Relat Res 478(7):1622-1633

25. Shohat N, Parvizi J (2017) Prevention of periprosthetic joint infection: examining the recent guidelines. J Arthroplasty 32(7):2040-2046. https://doi.org/10.1016/j.arth.2017.02.072

26. Slifka KJ, Yi SH, Reddy SC, Baggs J, Jernigan JA (2018) Theattributable mortality of prostheticjoint infection after primary hip and knee arthroplasty among medicare beneficiaries, 2005-2012. Open Forum Infect Dis 5(Suppl 1):118

27. Springer BD, Cahue $S$, Etkin $C D$, Lewallen DG, McGrory BJ (2017) Infection burden in total hip and knee arthroplasties: an international registry-based perspective. Arthroplast Today 3(2):137-140. https://doi.org/10.1016/j.artd. 2017.05.003

28. Statistisches Bundesamt (Destatis) http://wwwgenesis.destatis.de/genesis/online. Zugegriffen: 25. Dez. 2020

29. Tan TL, Rajeswaran $H$, Haddad $S$, Shahi $A$, Parvizi J (2016) Increased risk of periprosthetic joint infections in patients with hypothyroidism undergoing total joint arthroplasty. J Arthroplasty 31(4):868-871. https://doi.org/10.1016/j.arth. 2015.10.028

30. Tande AJ, Patel R (2014) Prosthetic joint infection. Clin Microbiol Rev 27(2):302-345

31. Tritt $K$, von Heymann F, Zaudig M, Zacharias I, Söllner W, Loew T (2008) Entwicklung des 
Fragebogens „ICD-10-Symptom-Rating“ (ISR) [Development of the "ICD-10-Symptom-Rating" (ISR) questionnaire].ZPsychosom Med Psychother 54(4):409-418. https://doi.org/10.13109/zptm. 2008.54.4.409

32. Winkler T, Stuhlert MGW, Lieb E, Müller M, von Roth $P$, Preininger $B$ et al (2019) Outcome of short versus long interval in two-stage exchange for periprosthetic joint infection: a prospective cohort study. Arch Orthop Trauma Surg 139(3):295-303. https://doi.org/10.1007/s00402-018-3052-4

33. Xiong J, Lipsitz O, Nasri F, Lui L, Gill H, Phan L et al (2020) Impact of COVID-19 pandemic on mental health in the general population: A systematic review. J Affect Disord 277:55-64. https://doi.org/ 10.1016/j.jad.2020.08.001

\section{Knie Journal}

\section{Primär maligne Knochentumoren} am Kniegelenk

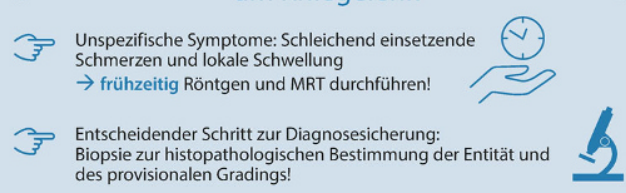

Cave: Bei unsachgemäßer Biopsie und Resektion kann durch Kontamination von umliegendem Gewebe eine Amputation erforderlich werden!

Das Tumorstaging umfasst Entität, Grading, Ausdehnung und Metastasen und erlaubt die interdisziplinäre Ableitung von Behandlungsstrategie und Prognose. Die Therapie des Osteo- und Ewing-Sarkoms besteht aus neo-
und adjuvanter Chemotherapie sowie weiter Tumorresektion.

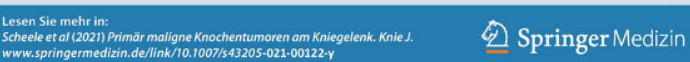

Primär maligne Knochentumoren sind äußerst selten, treten aber, in Abhängigkeit der genauen Entität, gehäuft in anatomischer Nähe zum Kniegelenk auf.

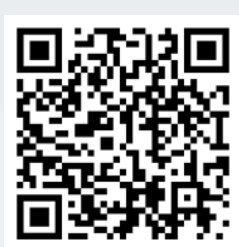

Lesen Sie weiter unter

www.springermedizin.de/link/

10.1007/s43205-021-00122-y

oder nutzen Sie den QR-Code.

Diesen und weitere spannende Beiträge aus Ihrem Fachgebiet lesen Sie mit unserem Online-Abo e.Med. Dieses bietet Ihnen u.a. Volltextzugriff auf alle Zeitschriften Ihres Fachgebiets wie z.B. Der Unfallchirurg, Der Orthopäde, Obere Extremität, Arthroskopie, Operative Orthopädie \& Traumatologie, Knie Journal und viele weitere. Testen Sie das Angebot 14 Tage kostenfrei: www.SpringerMedizin.de/eMed. 